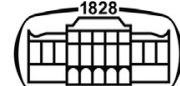

AKADÉMIAI KIADÓ

UNIVERSITY of DEBRECEN

\section{International Review of Applied Sciences and Engineering}

13 (2022) 1, 63-69

DOI:

$10.1556 / 1848.2021 .00308$

(c) 2021 The Author(s)

\section{ORIGINAL RESEARCH} PAPER

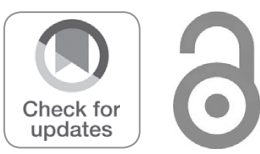

Corresponding author.

E-mail: spnz2014@gmail.com

\title{
Realization of fractional band pass filter on reconfigurable analog device
}

\author{
Sunil Narayan* (-), Utkal Mehta, Rita Iro, Hilda Sikwa'ae, \\ Kajal Kothari and Nikhil Singh
}

School of Information Technology, Engineering, Mathematics, and Physics, The University of the South Pacific, Laucala Campus, Suva, Fiji

Received: May 2, 2021 • Accepted: July 18, 2021

Published online: August 31, 2021

\section{ABSTRACT}

This paper presents a realization of fractional-order Band pass-filter (FOBF) based on the concepts of fractional order inductors and fractional order capacitors. The FOBF is designed and implemented using both simulation and hardware approaches. The proposed filter order is considered up to second order or less with any real positive number. One of the cases is considered when $\alpha \leq 1$ and $\beta \geq 1$. In the second case, the filter is designed when $\beta \leq 1$ and $\alpha \geq 1$. In order to calculate the optimal filter parameters, the modified Particle Swarm Optimization (mPSO) algorithm has been utilized for coefficient tuning. Also, a generalized approach to design any second order FOBF is discussed in this work. The realization and performance assessment have been carried out in simulation environment as well as in lab experiment with field programmable analog array (FPAA) development board. The experimental results indicate the value of efforts to realize the fractional filter.

\section{KEYWORDS}

fractional order, band pass filter, optimization, FPAA, quality factor and peak frequency

\section{INTRODUCTION}

In the last decade, Applied Mathematics with fractional calculus has become popular among researchers in different disciplines of science, technology and engineering. Fractional calculus provides an excellent instrument for the description of memory and hereditary properties of various materials and processes [1]. A fractional-order model or transfer function has better flexibility in comparison to classical integer order model, in which system dynamics are not taken into account. The review of fractional order modeling techniques and successfully obtained results in mathematical ways was presented for quick understanding of the research topic [2].

The mathematicians, Fourier, Euler, Laplace are among the many that fiddled with fractional calculus and the mathematical consequences. Many found definitions that fit the concept of a non-integer order integral or derivative using their own notations and methodologies. Nevertheless, the most famous but, also, most complex of them owed to RiemannLiouville and Grunwald-Letnikov are given below to start the understanding of fractional derivation and its physical interpretation [1-7].

The Riemann-Liouville [RL] definition of a fractional derivative is given by

$$
\frac{d^{\alpha}}{d t^{\alpha}} f(t) \equiv D^{\alpha} f(t)=\frac{1}{\Gamma(1-\alpha)} \frac{d}{d t} \int_{0}^{t}(t-\tau)^{-\alpha} f(\tau) d \tau,
$$

where $0<\alpha<1$. The Grunwald-Letnikov (GL) approximation in a more physical interpretation of a fractional derivative is given by

$$
D^{\alpha} f(t) \equiv(\Delta t)^{-\alpha} \sum_{j=0}^{m}(-1)^{j} n_{j}^{\alpha} f((m-j) \Delta t),
$$


where $\Delta t$ is the integration step and $n_{j}^{\alpha}=(-1)^{j}(\Gamma(j-\alpha) /$ $(\Gamma(1-\alpha) \Gamma(j+1)))$.

Applying the Laplace transform to (1) yields,

$$
L\left\{d_{t}^{\alpha} f(t)\right\}=s^{\alpha} F(s) .
$$

It is therefore possible to define a fractance element as one whose impedance $Z$ is proportional to $s^{\alpha} ; \alpha$ is arbitrary. It is noted that though the fractional derivatives are being applied to the various areas in science and engineering, yet it is vital to know the key value of each definition in application fields. The fractional calculus results have been observed in various fields such as control design [8-12], electrical circuits [13], stability analysis [14], mechanics [15], electromagnetic [16, 17], and bioengineering [18, 19] and progress is ongoing to date.

While designing the analog frequency filters traditionally of integer-order, it can also be designed using fraction order. In particular, as per work presented in the paper, the purpose of fractional filter designed to estimate the ideal response behavior for any specified performance filter. The band pass filter is basically a frequency selective circuit that accepts or rejects signals of a particular frequency band [20]. The design of any analog filter needs to determine the transfer function coefficients that will yield the desired filter characteristics, i.e., magnitude, phase, or delay.

A wide range of different design procedures are used on approximating interger order filter magnitude response (Butterworth [3], Chebyshev [4], Inverse-Chebyshev [5], Elliptic [6], arbitrary quality factor [7]) by the fractionalorder filter transfer function (FOTF). These approaches are successful and more works are largely being done on lowpass (LP) and high pass (HP) filters while little attention is given to other critical filters such as band pass, notch and comb filters. It has been found that fractional LP and HP filters of order $(1+\alpha)$ have been achieved and tested practically with minimal errors in passband response, accurate $-3 \mathrm{~dB}$ frequency response and greater stability margin [8].

As per theoretical operation, the band pass filters accept and rejects signals of a particular frequency band in a basic frequency selective circuit $[8,12]$ to provide narrow band characteristic. The filter is required a high-quality factor $(\mathrm{Q}$ factor). The high $\mathrm{Q}$ band pass filter can be used in power system engineering to reduce harmonic voltage and current distortions [13-16], the design of wireless transmitters and receivers, audio signal processing, seismology, optics, instrumentation, sonar and many more [9, 21, 22]. Literature presented using harmonic current injection, use of controllable reactors in place of fixed reactors, and direct current ripple injection to improve the performance of band pass filters $[17,18]$.

In this work, the desired characteristic of the parameters varies from the designed value that can be always be maintained by computing the coefficient and real value differential orders. Firstly, the modified particle-swarmoptimization ( $\mathrm{mPSO}$ ) is used to optimize values of the filter in order to satisfy the constraint requirements. The other optimization procedures compared with $\mathrm{MPSO}$, the advantages of the mPSO are that it is easy to implement with few parameters needing adjustment. The performance of the new fractional band pass filters has been verified. Secondly, the optimum order of approximation $s^{\alpha}$ is proposed in order to implement the fractional differentiator in hardware with acceptable accuracy, and the resulting filter is implemented in the Anadigm development environment of FPAA. The waveforms from the proposed FBPF are measured with various ranges of signal input frequencies. The performance generalized approach to design fractional-order band pass filter of orders ' $\alpha$ ' and ' $\beta$ ' respectively as $(\alpha \leq 1$ and $\beta \geq 1)$ and $(\beta \leq 1$ and $\alpha \geq 1)$ has been studied and compared with corresponding integer filters through both experimentation and simulation. The obtained results confirm that the actual fractional filters behavior closely follows the theoretical approximations for all values of $\alpha$.

The paper is organised as follows: Section 2 introduces fractional order BP transfer functions and finding the coefficients using modified Particle Swam Optimization (mPSO). Section 3 describes the implementation of the results using different values for $\alpha$ and $\beta$. Section 4 discusses the hardware implementation of fractional band pass filter using reconfigurable analog device.

\section{FRACTIONAL ORDER BAND PASS FILTER TRANSFER FUNCTIONS}

The realization of first order fractional capacitor (FC) and fractional inductor (FI) is important to obtain the transfer functions of fractional order (FO) for second order Band Pass filter (BPF). The FC and FI are known as fractional order elements (FOE) and they are dependent on the value of exponent. The order of the FC depends on $\beta$ and the order of FI depends on $\alpha$. The circuit realization in Fig. 1 shows how to obtain the fractional order BPF with the resistance, FC and FI which are connected in series.

$$
Y(s)=\frac{I(s)}{V(s)}=\frac{\frac{s^{\beta}}{L_{F}}}{s^{\alpha+\beta}+\left(\frac{R}{L_{F}}\right) s^{\beta}+\left(\frac{1}{C_{F} L_{F}}\right)}
$$

Equation (4) shows the admittance of the circuit which will be the transfer function of the fractional BPF. The coefficients $k_{1}, k_{2}$ and $k_{3}$ are the parameters of the transfer function (4) and they are obtained by using the optimization algorithm technique called mPSO. The BPF transfer function with the FC and FI orders, they give attenuations in

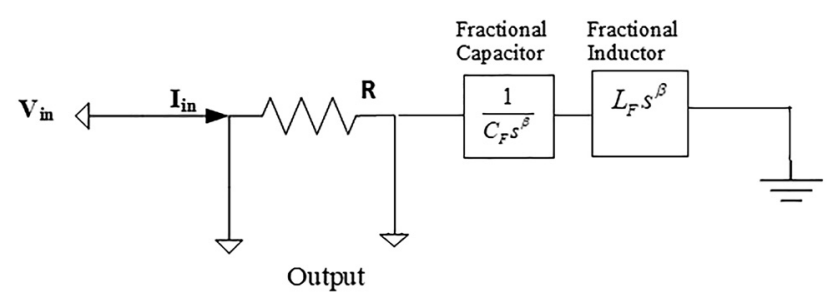

Fig. 1. Fractional-order band pass filter 
stop-band of $-20 \alpha \mathrm{dB} /$ decade and $+20 \beta \mathrm{dB} /$ decade for frequencies that are higher and lower than the center frequency. Therefore, the second order BPF transfer function for Butterworth filter provides more flexibility and a lower order than that of its integer order counterparts.

\subsection{Optimization of FO BPF transfer function coefficients using mPSO}

The transfer function obtained in (1) for the BPF response, its new coefficients are obtained using the mPSO optimization algorithm. Using this algorithm, the new optimized

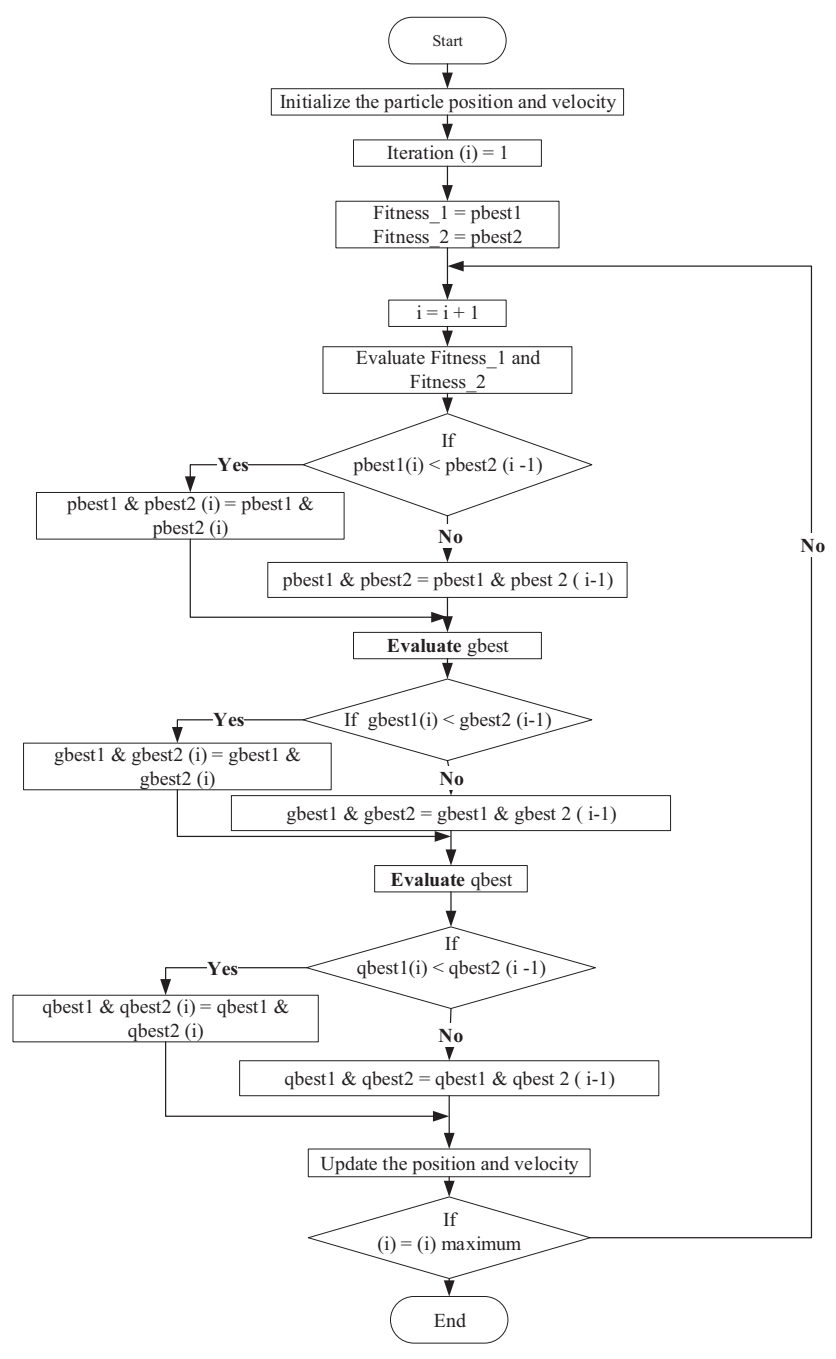

Fig. 2. Flow Chart of the bi-level PSO algorithm coefficients $k_{1}, k_{2}$ and $k_{3}$ parameters can be determined for desired filter that is required as per objective. $\mathrm{k}_{1}, \mathrm{k}_{2}$ and $\mathrm{k}_{3}$ inputted as variables to be optimized using mPSO [8]. For the $\mathrm{BPF}$, the optimization carried out for the objective function between the $-3 \mathrm{~dB}$ close to $1 \mathrm{rad} \mathrm{s} \mathrm{s}^{-1}$ and Least Square Error (LSE) is solved to vary it close to 1 .

Minimum LSE, calculated as:

$$
|E c(j \omega)|=\sum_{i=1}^{n}\left[\left|H_{B P}\left(k, \omega_{i}\right)\right|-\left|H_{1+\alpha}\left(\omega_{i}\right)\right|\right]^{2}
$$

$-3 \mathrm{~dB}$ frequency close to $1 \mathrm{rad} \mathrm{s}^{-1}$ by minimization of:

$$
\left(\omega_{3 d B}-1\right)^{2}
$$

From the mPSO algorithm flow chart in Fig. 2 below, it is explained how the set of optimal coefficients are obtained for the transfer function order $(\alpha+\beta)$. Here, pbest 1 , pbest 2 and pbest 3 are said to be the best positions for particles 1 , 2 and 3 revering to the objective function called in the flow diagram. Whilst for the gbest1, gbest 2 and gbest 3 it simply refers to global best position of particles 1,2 and 3 respectively. $\mathrm{H}_{1+\alpha}$ is the FO BPF of order $(\alpha+\beta)$ which needs to be approximated by $\mathrm{H}_{\mathrm{BP}}$ and $\mathrm{k}$ is the coefficients $\left(k_{1}, k_{2}, k_{3}\right)$ of FO BPF function given in (4).

Therefore, in this paper we have used mPSO algorithm for more computable efficiency and it is adaptable for optimization of bi-level objective functions. Thus, mPSO optimization improves the desired filter characteristics and provides a lower LSE value and high stability margin. There are generalized equations for different types of filters with stability constraints and optimization but for the one used in this work is FO band pass Butterworth filter. This paper also proposes the best designed method for the FO for BPF. The optimal new coefficient values are obtained from a bi-level optimization that approximates the passband of the Butterworth response with fractional-step stopband attenuation in Table 1.

\section{RESULTS AND VALIDATION}

\subsection{Optimized values for $k$ coefficients}

The given coefficients are optimized using the mPSO algorithm for different $\alpha$ and $\beta$. After optimizing the new coefficients for $\mathrm{k}_{1}, \mathrm{k}_{2}$ and $\mathrm{k}_{3}$ are obtained from curve fitting tools in MATLAB. The optimized coefficients are plotted to see the magnitude and phase response as shown in Fig. $3 a$ and b.

Table 1. Values of the $k$ coefficients

\begin{tabular}{ll}
\hline Parameter specifications \\
\hline Given coefficients & Optimized coefficients from PSO Algorithm \\
\hline$k_{1}=13888.89$ & $k_{1}=1.40 \times 10^{4}+78.24 \alpha+154.50 \beta-84.41 \alpha^{2}-182.10 \alpha \beta-174.20 \beta^{2}+122.70 \alpha^{2} \beta+135.30 \alpha \beta^{2}+15.06 \beta^{3}$ \\
$k_{2}=13888888.89$ & $k_{2}=1.9 \times 10^{7}+1.37 \times 10^{5} \alpha+2.44 \times 10^{5} \beta+2.99 \times 10^{4} \alpha^{2}-3.95 \times 10^{5} \alpha \beta-6.52 \times 10^{4} \beta^{2}-3.33 \times 10^{4} \alpha^{2} \beta$ \\
& $\quad+1.51 \times 10^{5} \alpha \beta^{2}+579.30 \beta^{3}$ \\
$k_{3}=734861846$ & $k_{3}=7.29 \times 10^{8}-4.63 \times 10^{6} \alpha-2.64 \times 10^{6} \beta+5.07 \times 10^{6} \alpha^{2}+9.36 \times 10^{6} \alpha \beta+1.57 \times 10^{6} \beta^{2}-8.17 \times 10^{6} \alpha^{2} \beta$ \\
& $\quad-1.53 \times 10^{6} \alpha \beta^{2}-1.32 \times 10^{5} \beta^{3}$ \\
\hline
\end{tabular}




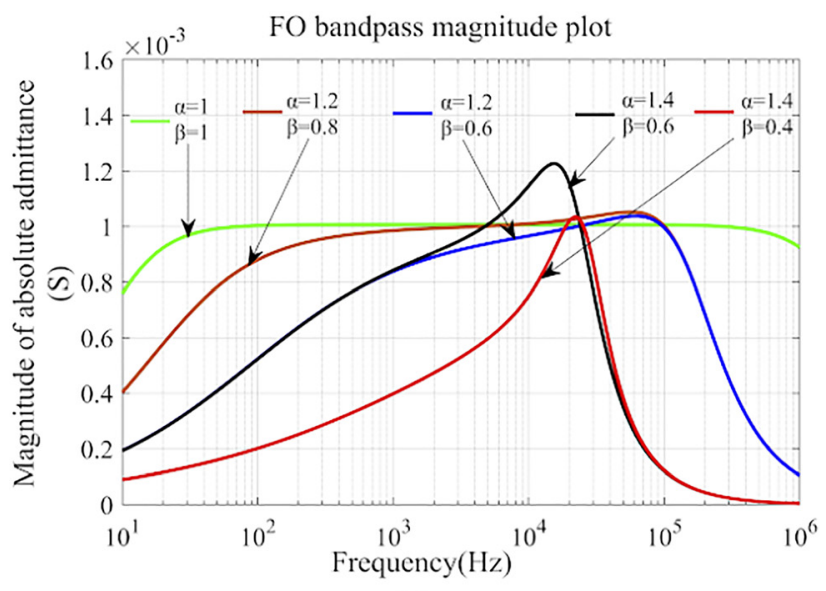

(a)

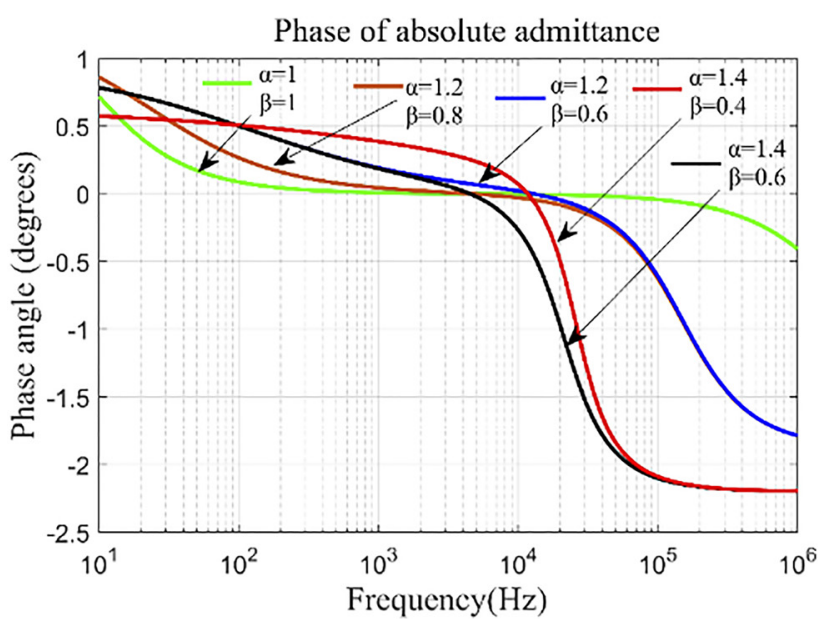

(b)

Fig. 3. (a). The magnitude plot for $\alpha \geq 1$ and $\beta \leq 1$ (b). Phase plot for $\alpha \geq 1$ and $\beta \leq 1$

\subsection{Simulation output results}

The optimized coefficients are then plotted in MATLAB to see the absolute admittance response of the FO BPF for different values of $\alpha$ and $\beta$. There were two different cases done for the simulations. Figure $3 \mathrm{a}$ and $3 \mathrm{~b}$ show the magnitude and phase plot for the case where $\alpha \geq 1$ and $\beta \leq 1$. Figures 3 and 4 also illustrate the graph for integer $(\alpha=1$ and $\beta=1)$ and non-integer BPFs, and observed that the non-integer graph has a sharper cut off compared to the integer filter.

The second case shows the simulations for $\alpha \leq 1$ and $\beta \geq$ 1 , and shows the magnitude and the phase plots as in Fig. 4, the frequency which the magnitude of admittance is maximum is not the same with the frequency at which the phase is zero. Thus for more flexibility and tuning characteristics the first case $(\alpha \geq 1$ and $\beta \leq 1)$ can be more adjusted to have sharper tuning than the second case $(\alpha \leq 1$ and $\beta \geq 1)$.

\subsection{Quality factor}

To determine the sharpness of tuning for the FO BPF, the quality factor is calculated by finding the poles of the fractional BPF. Using equation the poles of the transfer function

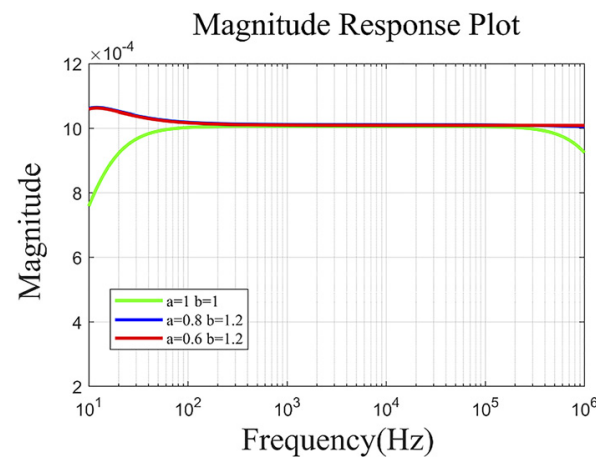

(a)

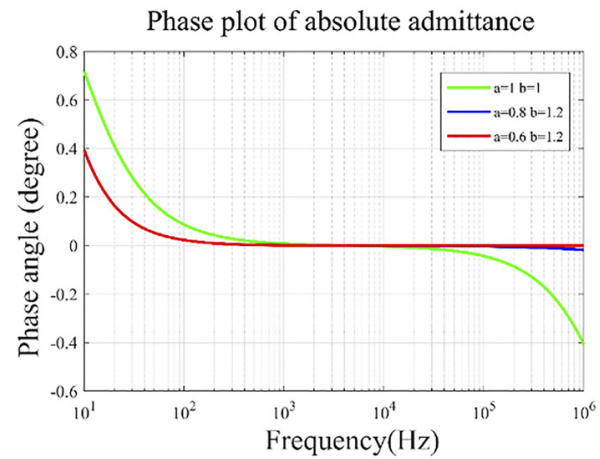

(b)

Fig. 4. (a). Magnitude plot for $\alpha<1$ and $\beta>1$. (b) phase plot of $\alpha<1$ and $\beta>1$

is calculated and it brings the pair of poles closest to the stability boundary of $\left(\left|\theta_{W}\right|=\frac{\pi \alpha}{2}\right)$. The pole of the FO BPF is in Eq. (4) where $W=s^{\beta}$ [19]. The approach to calculate the Q factor of this analysis was proposed in [19]. The simulation of Q factor is shown in Fig. 4, where the Q factor of integer order has the lowest sharpness tuning compared to those filter of non-integer. Figure 5 also shows the variation of the Q factor with the order of FC for $\alpha \geq 1$ and $\beta \leq 1$ with $\alpha+\beta=2$ where the $\mathrm{Q}$ factor increases as the order of FC and $\beta$ decreases that of FI $\alpha$ increases. The advantage of fractional order is that the $\mathrm{Q}$ factor may increase by changing the fractional exponents and keeping other parameters constant.

$$
W^{\frac{\alpha+\beta}{\alpha}}+k_{2} W+k_{3}=0
$$

Peak frequency $\left(\omega_{n}\right)$ is the frequency where maximum admittance is offered by FO BPF [23, 24]. The peak frequency of the FO BPF is calculated using Eq. (8). Peak frequency is the frequency at which the maximum admittance is offered from the magnitude of absolute admittance plot in Fig. 6 .

$$
\begin{aligned}
& \omega_{n}^{2(\alpha+\beta)}+\frac{p}{2 L_{F}^{2}} \omega_{n}^{\alpha+2 \beta}-\frac{q \beta}{2 \alpha L_{F}^{2}} \omega_{n}^{\beta}+\frac{r(\alpha-\beta)}{2 \alpha L_{F}^{2}} \omega_{n}^{\alpha+\beta}-\frac{\beta}{2 \alpha L_{F}^{2} C_{F}^{2}} \\
& \quad=0
\end{aligned}
$$

The results in Fig. 6 show the peak frequency for different values of $\beta$ values. The graph also shows that if $\beta=$ 1 the peak frequencies were lower that of those $\beta$ values less 


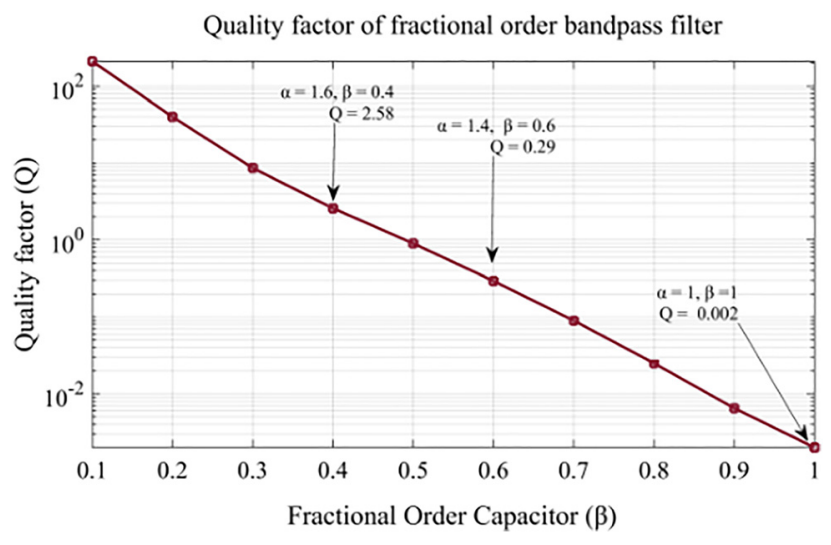

Fig. 5. Quality factor of the optimized parameters with different values of alpha and beta

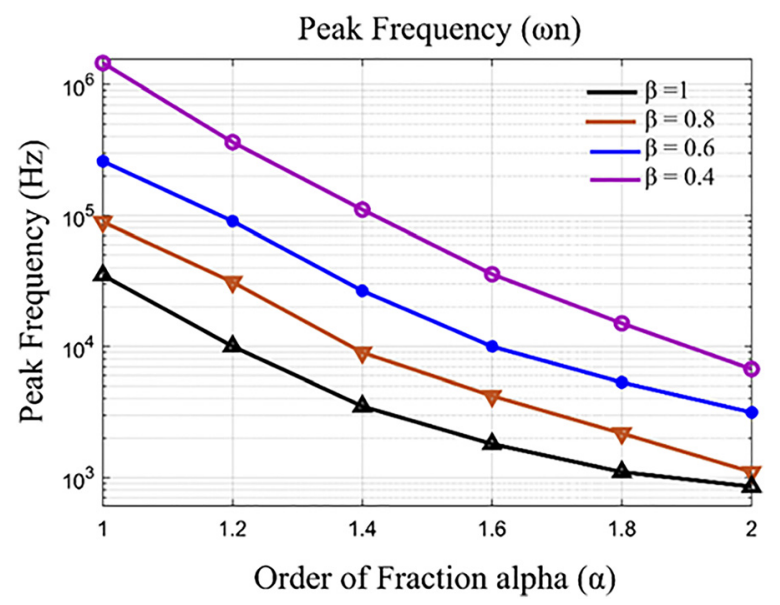

Fig. 6. The peak frequency response from the optimized magnitude plot

than 1. It is also seen that peak frequencies for $\beta$ values decrease with the increase in the order of $\alpha$. Thus, the advantage of FO BPF is that it has more flexibility in selection of peak frequency compared to integer order.

\section{HARDWARE IMPLEMENATION AND RESULTS}

The implementation of FO BPF on the FPAA development board of the overall set up diagram is shown in Fig. 7. The FPAA will interface between the single to differential converter and the differential to single converter with the input signal coming from the spectrum analyzer. The implementation uses an Anadigm Designer 2 development environment. This method uses the transfer function of pole and zero frequencies and the quality factor to realize the BPF $[9,19,20]$. The transfer function in Eq. (9) is then decomposed into the first and second order using two biquadratic filter CAM modules to approximate the fractional BPF. The FO BPF was realized and implemented on the FPAA

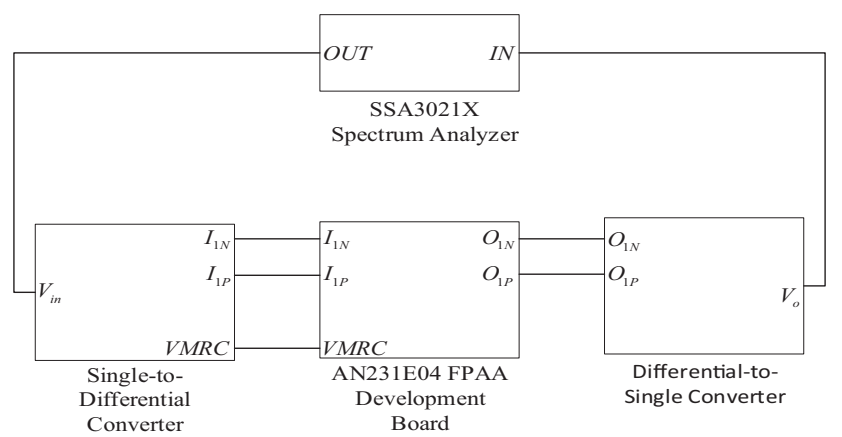

Fig. 7. The setup for the implementation of fractional band pass filter

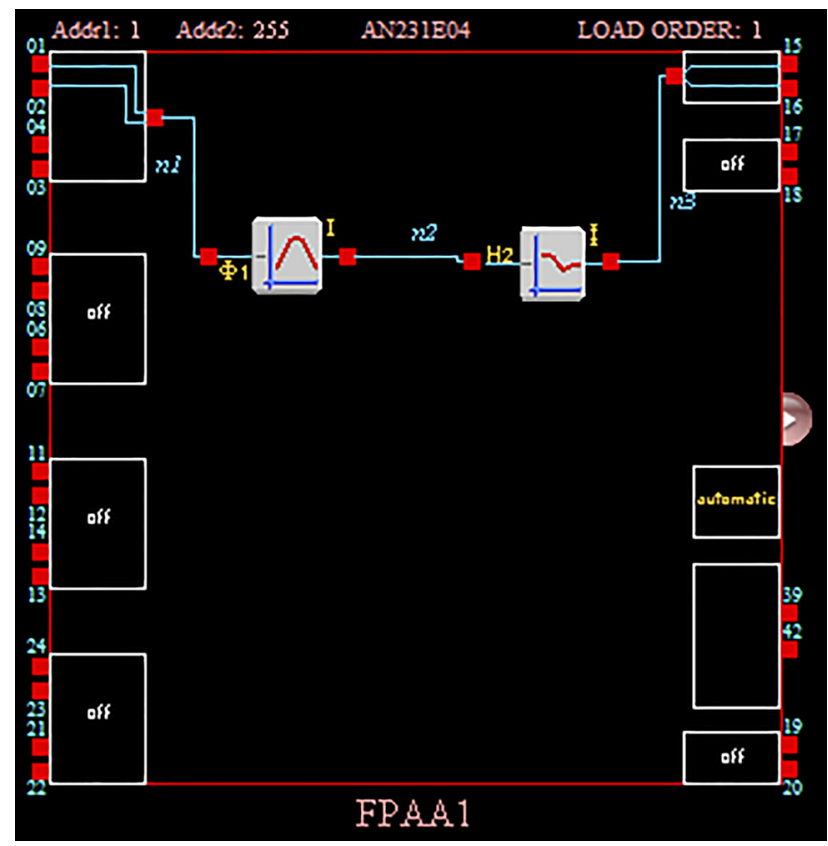

Fig. 8. (a). Anadigm designer

development board and the setup of the hardware experiment is shown below in Fig. 10. After implementing the FO BPF into the Anadigm Designer and interfaced with the FPAA development board the output was observed from the spectrum analyzer as shown in Fig. 11.

$$
H(s)=H_{1}(s) \times H_{2}(s)=\frac{1}{s^{2}+s+d_{o}} \times \frac{e_{o} s^{2}+e_{1} s+e_{2}}{s^{2}+d_{1} s+d_{2}}
$$

The connection of the two CAM is shown in Fig. 8 below in the Anadigm Designer.

In the Anadigm Designer the CAMs have different transfer functions for different filters, thus the resulted equations for the CAMs are as follows;

$$
\begin{gathered}
H(s)=T_{1}(s) \times T_{2}(s) \\
T_{1}(s)=-\frac{2 \pi f_{1} \frac{G_{1}}{Q_{1}} s}{s^{2}+\frac{2 \pi f_{1}}{Q_{1}}+4 \pi^{2} f_{1}^{2}}
\end{gathered}
$$




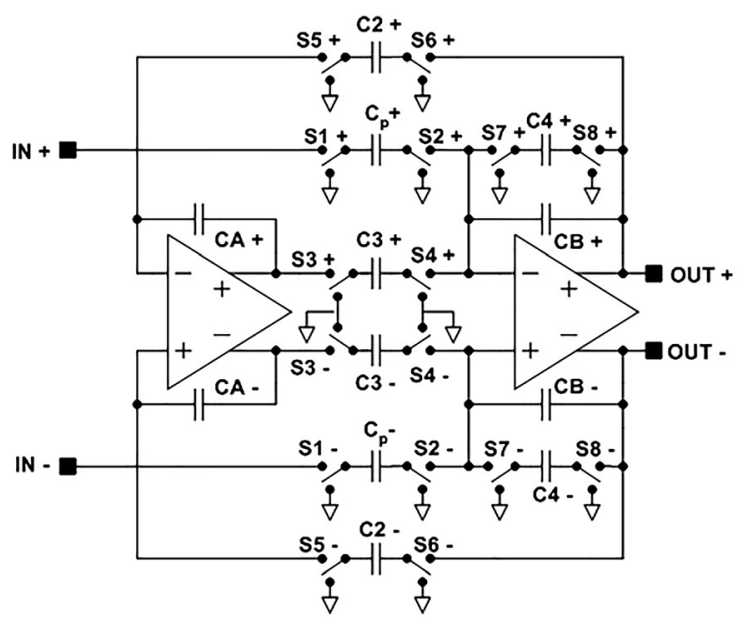

(a)

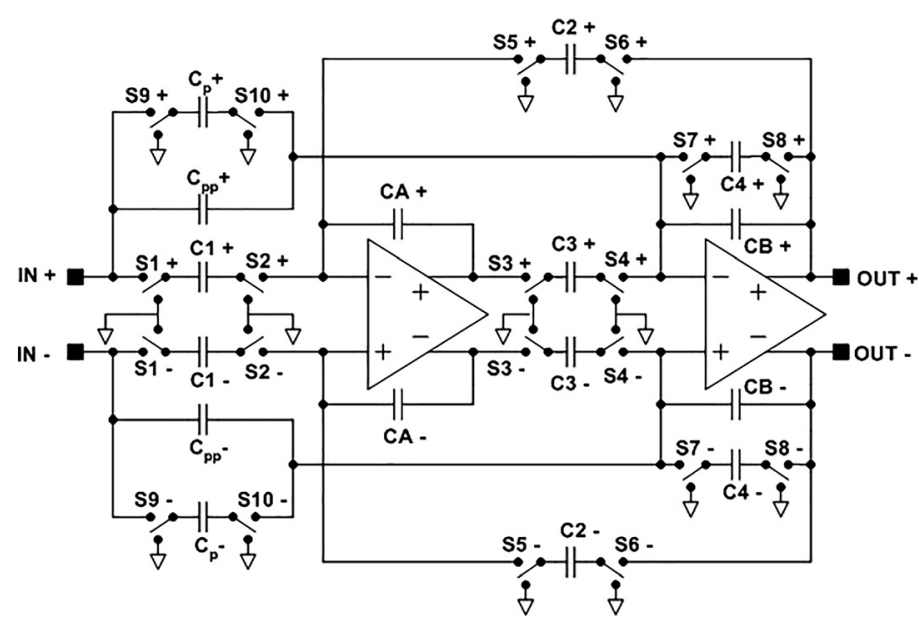

(b)

Fig. 9. Internal switched capacitor circuit to realize (a) band pass filter biquadratic CAM (b) pole and zero frequency biquadratic CAM

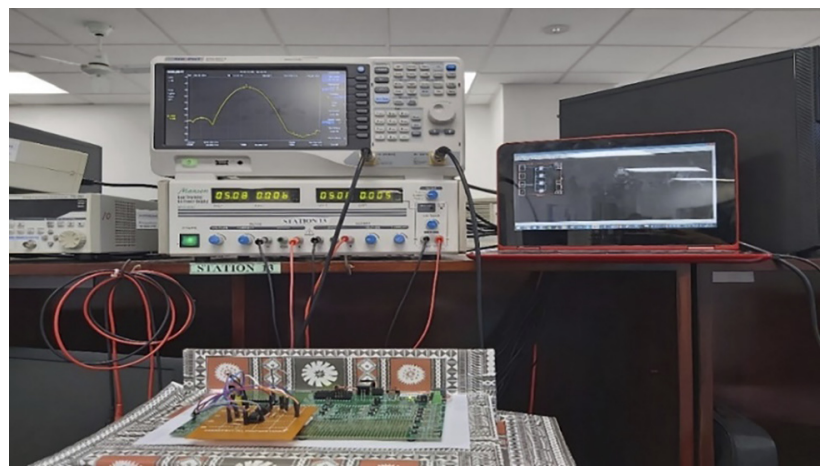

Fig. 10. The hardware setup

$$
T_{2}(s)=-\frac{s^{2}+\frac{2 \pi f_{2 z}}{Q_{2 z} s}+4 \pi^{2} f_{2_{z}}^{2}}{s^{2}+\frac{2 \pi f_{2 p}}{Q_{2 p}}+4 \pi^{2} f_{2_{p}}^{2}}
$$

where $\mathrm{T}_{1}$ is the transfer function of Biquadratic CAM with the option of band pass filter and $\mathrm{T}_{2}$ is the transfer function of the biquadratic CAM for the poles and zeros frequency. The transfer functions are realized by the switched capacitor technology as shown in Fig. 9.

The fractional-order band pass filter was realized and implemented on the FPAA development board and the setup of the hardware experiment is shown below.

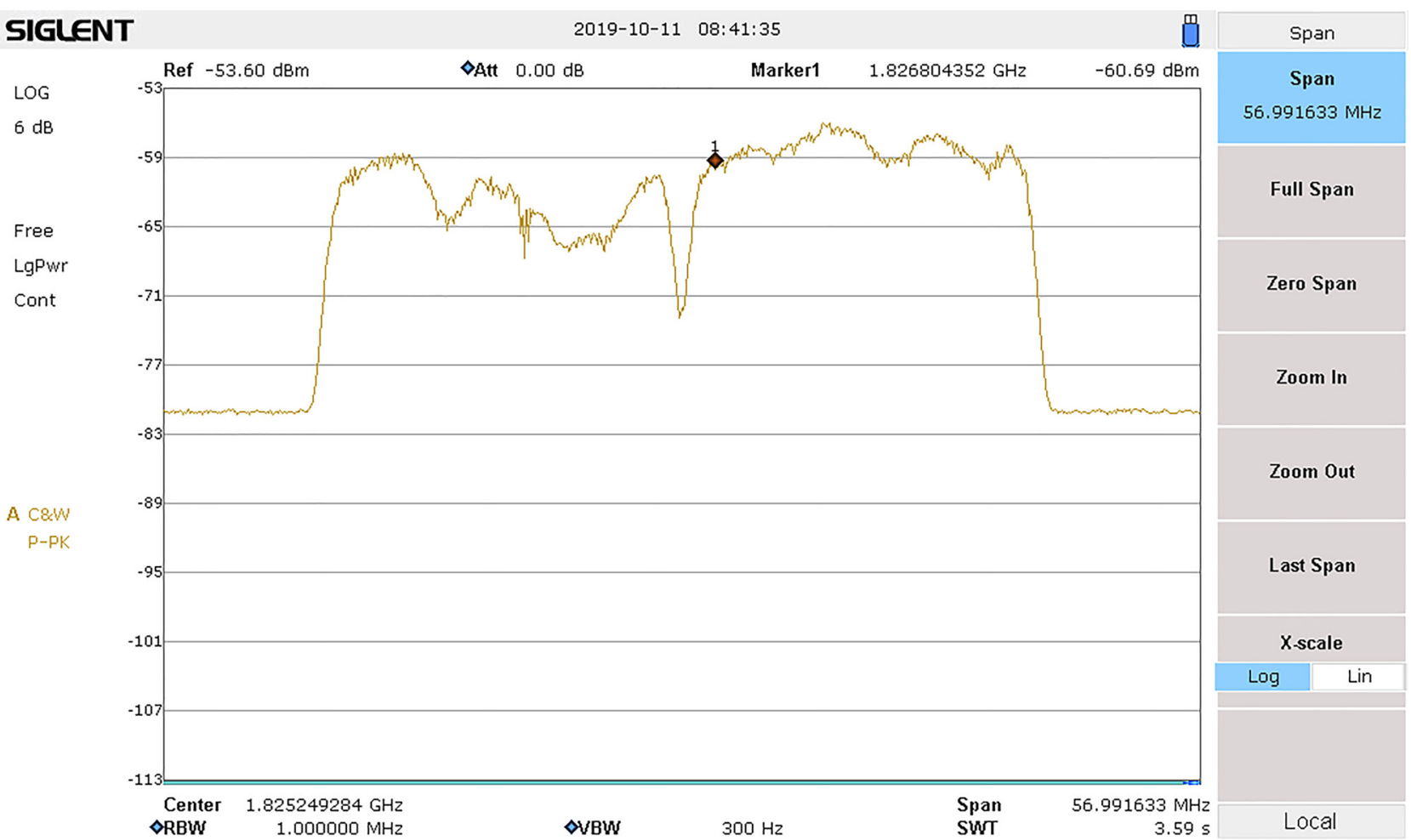

Fig. 11. The FO band pass output observed from spectrum analyzer 


\section{CONCLUSION}

The paper focused on the design and implementation of the fractional-order band pass filter with the practical realization using reconfigurable analog device. Different fractional-order exponents of $(\alpha, \beta)$ have been realized in this paper for $0<\alpha \leq 1$. The performance of design fractional-order filter by varying the values for $\alpha$ and $\beta$ were observed through simulation comparing with integer-order and hardware implementation on the reconfigurable analog device. The results showed that fractional-order band pass filter gives a flexibility to design the peak frequency by varying the values of ' $\alpha$ ' and ' $\beta$ ' within the fractional-order transfer function. Also observed from the results were the output waveforms of the proposed design band pass filter measured using the oscilloscope and the spectrum analyzer for different ranges of input frequency signal. In this way, the fractional-order filter of order $(\alpha+\beta)$ have been studied and compared with the corresponding integer order filter through simulation and experimentation.

\section{REFERENCES}

[1] R. Caponetto, G. Dongola, L. Fortuna, and I. Petráš, Factional Order System-Modeling and Control Applications. Singapore: World Scientific Publishing, 2010.

[2] K. Kothari, U. Mehta, and R. Prasad, "Fractional-order system modeling and its applications," J. Eng. Sci. Technol. Rev., vol. 12, no. 6, pp. 1-10, 2019.

[3] T. Freeborn, "Comparison of $(1 \mathrm{p} \alpha)$ fractional-order transfer functions to approximate lowpass Butterworth magnitude responses," Circuits Syst. Signal Process., vol. 35, no. 6, pp. 1983-2002, 2016.

[4] T. Freeborn, B. Maundy, and A. Elwakil, "Approximated fractional order Chebyshev lowpass filters," Math. Probl. Eng., vol. 2014, p. 832468, 2015.

[5] T. Freeborn, A. Elwakil, and B. Maundy, "Approximated fractional-order Inverse Chebyshev lowpass filters," Circuits Syst. Signal Process., vol. 35, no. 6, pp. 1973-82, 2016.

[6] T. Freeborn, D. Kubanek, J. Koton, and J. Dvorak, "Fractionalorder lowpass elliptic responses of $(1+\alpha)$-order transfer functions," in 2018 41st International Conference on Telecommunications and Signal Processing (TSP). IEEE, 2018.

[7] D. Kubanek and T. Freeborn, " $(1+\alpha)$ fractional-order transfer functions to approximate low-pass magnitude responses with arbitrary quality factor," Int. J. Electron. Commun., vol. 83, pp. 570-8, 2018.

[8] N. Singh, U. Mehta, K. Kothari, and M. Cirrincione, "Optimized fractional low and highpass filters of $(1+\alpha)$ order on FPAA," Bulletın Polish Acad. Sci. Tech. Sci., vol. 68, no. 3, pp. 635-44, 2020.
[9] A. Soltan, A. G. Radwan, and A. M. Soliman, "Fractional order filter with two fractional elements of dependant orders," Microelectron. J., vol. 43, no. 11, pp. 818-27, 2012.

[10] V. Duarte and J. Costa, "Time-domain implementations of noninteger order controllers," in Proc. Controlo, Portugal, Sept. 5-7, 2002, pp. 353-8.

[11] A. Radwan, A. Elwakil, and A. Soliman, "Fractional-order sinusoidal oscillator: Design procedure and practical examples," IEEE Trans. Circuits Syst., vol. 55, no. 7, pp. 2051-63, Jul. 2008.

[12] A. Radwan, "Stability analysis of the fractional-order RLC circuit," J. Fract. Calc. Appl., vol. 3, 2012.

[13] J. Baranowski and P. Piatek, "Fractional band-pass filters: Design, implementation and application to EEG signal processing," $J$. Circuits, Syst. Comput., vol. 26, no. 11, pp. 1-21, 2017.

[14] A. Radwan, K. Moddy, and S. Momani, "Stability and nonstandard finite difference method of the generalized Chua's circuit," Comput. Math. Appl., vol. 62, pp. 961-70, 2011.

[15] T. Doehring, A. H. Freed, E. O. Carew, and I. Vesely, "Fractional order viscoelasticity of the aortic valve: An alternative to QLV," J. Biomech. Eng., vol. 127, no. 4, pp. 700-8, 2005.

[16] K. David, F. Todd, and K. Jaroslav, "Fractional-order band pass filter design using fractional characteristic specimen functions," Microelectronics J., vol. 86, pp. 77-86, 2019.

[17] M. C. Tripathy, D. Mondal, K. Biswas, and S. Sen, "Experimental studies on realization of fractional inductors and fractional-order bandpass filters," Int. J. Circuit Theor. Appl., vol. 43, no. 9, pp. 1183-96, 2015.

[18] L. A. Said, K. Biswas, and A. G. Radwan, "Fractional-order filter design," in Fractional-Order SystemEles. Elsevier Inc., 2018, pp. $357-81$.

[19] P. Ahmadi, B. Maundy, A. S. Elwakil, and L. Belostotski, "Highquality factor asymmetric-slope band-pass filters: a fractional-order capacitor approach," IET Circuits, Devices Syst., vol. 6, no. 3, p. 187, 2012.

[20] T. Freeborn, "Comparison of $(1+a)$ fractional-order transfer function to approximate low pass Butterworth magnitude response," Circuit Syst. Signal Process, vol. 35, no. 1, pp. 1983-2002, 28 December 2015.

[21] A. Soltan, A. G. Radwan, and A. M. Soliman, "CCII based fractional filters of different orders," J. Adv. Res., vol. 5, pp. 157-64, 2014.

[22] P. R. Hemavathy, Y. Mohamed Shuaib, and S. K. Lakshmanaprabu, "Internal model controller based PID with fractional filter design for a nonlinear process," Int. J. Electr. Comput. Eng. (IJECE), vol. 10, no. 1, pp. 243-54, February 2020.

[23] A. G. Radwan, A. S. Elwakil, and A. M. Soliman, "On the generalization of second order filters to the fractional order domain," J. Circuits, Syst. Comput., vol. 18, no. 2, pp. 361-86, 2009.

[24] T. Freeborn, B. Maundy, and A. Elwakil, "Field programmable analogue array implementation of fractional step filters," IET Circuits Devices Syst., vol. 4, no. 6, pp. 514-24, 2010. 